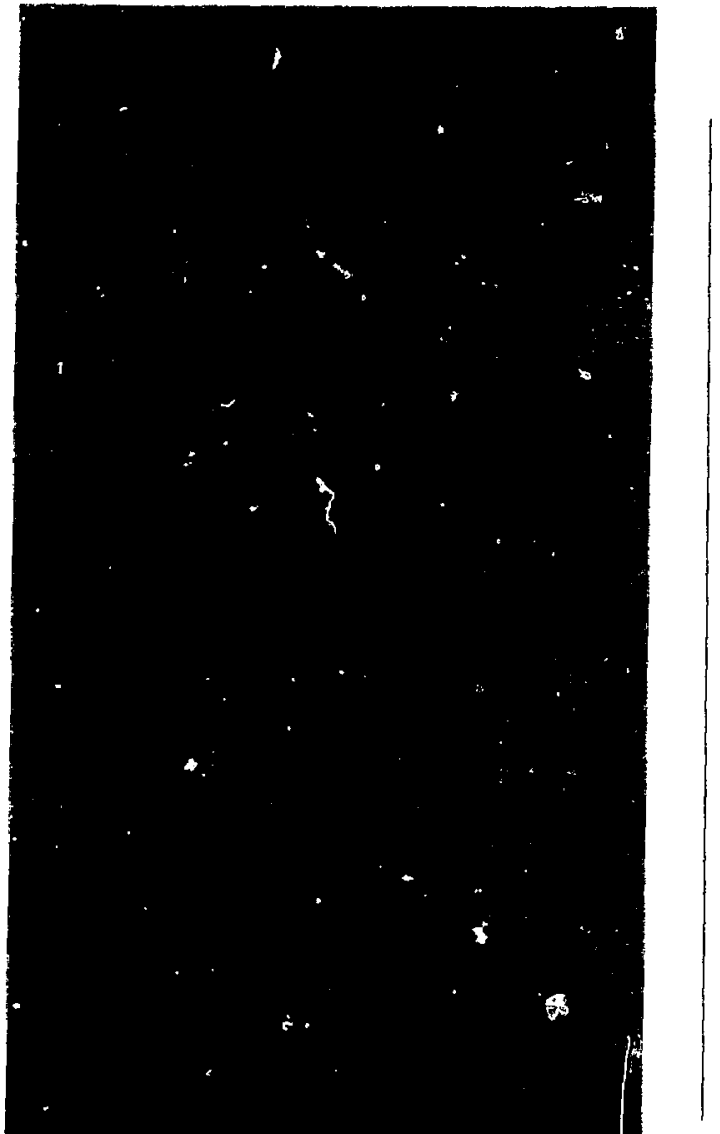

\title{
Center of Buoyancy Definition
}

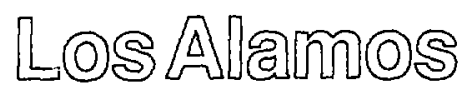

Los Alamos National Laboratory is operated by the Unizersity of California for the United States Department of Energy under contract W-7405-ENG-36. 
This work was supported by the US Department of Energy, Office of Energy Research.

An Affirmative Action/Equal Opportunity Employer

This report was prepared as an account of work sponsored by an agency of the United States Government, Neither the United States Government nor any agency thereof. nor any of their employees, makes any warranty, express or implied, or assumes any legal liability or responsibility for the accuracy, completeness, or usefulness of any information, apparalus, product, or process disclosed, or represents that its use would not infringe prizately owned rights. Reference herein to any specific commercial product, process, or service by trade name, trademark, manufucturer, or otherwise, does not necessarily constitute or imply its endorsement, recommendation, or favoring by the United States Government or any agency thereof. The ziews and opinions of authors expressed herein do not necessurily state or reflect those of the United Stales Government or any agency thereof. 


\section{Center of Buoyancy Definition}

\section{Vernon Sandberg}

\section{DISCLAIMER}

This report was prepared as an account of work sponsored by an agency of the United States Government. Neither the United States Government nor any agency thereof, nor any of their employees, makes any warranty, express or implied, or assumes any legal liability or responsibility for the accuracy, completeness, or usefulness of any information, apparatus, product, or process disclosed, or represents that its use would not inıringe privately owned rights. Reference herein to any specific commercial product, process, or service by trade name, trademark, manufacturer, or otherwise does not necessarily consti ute or imply its endorsement, recommendation, or favoring by the United States Government or any agency thereof. The views and opinions of authors expressed herein do not necessarily state or reflect those of the United States Government or any agency thereof. 


\title{
CENTER OF BUOYANCY DEFINITION
}

\author{
by \\ Vernon Sandberg
}

\begin{abstract}
The center of buoyancy of an arbitrary shaped body is defined in analogy to the center of gravity. The definitions of the buoyant force and center of buoyancy in terms of integrals over the area of the bedy are converted to volume integrals and shown to have simple intuitive interpretations.
\end{abstract}

Large water Cherenkov detectors for neutrino experiments require large volume photomultiplier tubes to be mounted under water. The mechanical forces and torques acting on the tubes can be substantial. To insure long tube life and to minimize mechanical failures attention must be paid as to how the tubes are mounted. To minimize torques on the tube envelope and the support frame requires a knowledge of the torques produced by the buoyant forces acting on the tube and the location of the ceriter of buoyancy. This note provides a careful definition of the center of buoyancy.

The buoyant force on a Burle $88545^{\prime \prime}$ diameter photomultiplier tube submerged under water is 4.5 lbs. For a Hamamatsu R $14088^{\prime \prime}$ diameter tube the buoyant force is $7 \mathrm{lbs}$ and for a Hamamatsu R1449 20" diameter tube the buoyant force is $91 \mathrm{lbs}$. With forces of these magnitudes careful attention needs to be paid as to the location and design of the mounting fixtures. If a tube is fastened to a fixnure, for example, at its pinout end a large torque (approximately $12 \mathrm{ft}$-lbs for a 5 "diameter tube) will be produced by the buoyant force that could damage the tube and unnecessarily strain the support structure. The problem is not to hold up the tubes, but to hold them down.

The optimal mounting position on the tube is at the location where the sum of the torques due to the buoyant force and weight of the tube vanish. The torque produced by the weight of the tube is equal to the weight times the perpendicular distance from the vertical line through the support point to the tube's center of gravity. If this were the only force involved (its weight) then supporting the tube at its center of gravity would produce zero torque; however, we are concerned with mounting the tube under water and we must take the much larger buoyant forces into consideration. By analogy with center of gravity we define the center of buoyancy to be that point about which the torques due to the buoyant force vanish. In this note we will precisely define what this means and derive the mathematical expressions for the buoyant force and the center of buoyancy. In what follows we neglect the weight of the tube since for the phototubes under consideration the weight is a small fraction of the buoyant force.

An arbitrary shaped body submersed in water will be in static equilibrium if the sum of the forces and the sum of the torques acting on it are zero. This means that the buoyant force $\mathrm{F}_{\mathrm{B}}$ must be equal in magnitude and opposite in 
direction to the external support force $\mathrm{F}_{\mathrm{exl}}$ that holds the body under water, namely:

$$
\overrightarrow{\mathrm{F}}_{\mathrm{ext}}=-\overrightarrow{\mathrm{F}}_{\mathrm{B}}
$$

The hydrostatic pressure at a depth $\mathrm{z}$ below the surface is given by

$$
\mathrm{p}=\mathrm{p}_{0}+\rho g z
$$

where $p_{0}$ is the surface (i.e., atmospheric) pressure, $\rho$ is the density of water, and $g$ is the acceleration due to gravity. The element of force $\overrightarrow{d F}_{B}$ on an element of area $\mathrm{d} \overrightarrow{\mathrm{a}}$ of the body is given by

$$
\mathrm{d} \overrightarrow{\mathrm{F}}_{\mathrm{B}}=p \mathrm{~d} \overrightarrow{\mathrm{a}}
$$

and the total buoyant force is given by the vector integral

$$
\vec{F}_{B}=\int_{\mathbf{a}} d \vec{F}_{B}=\int_{\mathbf{a}} p d \vec{a}
$$

This integral is nonzero due to the existence of a pressure gradient along the $z$-diection. The torque about an origin $\mathrm{O}$ due to the buoyant force is given by

$$
\vec{\Gamma}_{B}=\int_{\mathbf{a}} \vec{X} \times d \vec{F}_{B}
$$

where $\overrightarrow{\mathrm{X}}$ is the position vector to the point $(x, y, z)$. Let $\vec{A}$ denote the vector from the origin $O$ to the support point. Then $\vec{F}_{\text {ext }}$ acts at $\vec{A}$ to produce the support torque. To be in equilibrium this torque must balance the buoyant torque and we have for the center of buoyancy position vector $\vec{A}_{\mathrm{CB}}$ the condition

$$
0=\vec{\Gamma}_{B}+\vec{A}_{C B} \times \vec{F}_{\text {ext }} \text {. }
$$

Solving for $\vec{A}_{C B}$ we find for the projection perpendicular to $\vec{F}_{\text {ext }}$ the vector $\vec{A}_{\perp}$

$$
\vec{A}_{\perp}=\left|\vec{F}_{B}\right| 2 \vec{A}_{C B}-\vec{F}_{B}\left(\vec{A}_{C B} \cdot \vec{F}_{B}\right)=\vec{F}_{B} \times \int_{a} \vec{X} \times d \vec{F}_{B}
$$

Only the perpendicular component is determined since any component of the support vector that is parallel to the buoyant force (or external force, remember we are assuming equilibrium) will not produce a torque. This expression is not particularly illuminating and can be reduced to a more convenient form by converting the area integrals into volume integrals over the volume of the body. This conversion is described in the appendix. Below we summarize the final expressions for the buoyant force and center of buoyancy in their integral-overvolume forms to relieve the reader of the burden of following the manipulations described in the appendix.

The buoyant force is given ty 


$$
\vec{F}_{B}=\rho g V \hat{z}
$$

where $\mathrm{V}$ is the volume bounded by area $\mathrm{a}$. This is recognized as Archimedes' principle: The buoyant force is equal to the weight of the volume of water displaced by the body.

The center of buoyancy is given by

$$
\vec{A}_{C B}=\frac{1}{V} \int_{V} \vec{X} d^{3} x
$$

Notice that this expression is equal to that for the center of gravity of a body of the same shape and size but with a constant unit density.

\section{APPENDIX}

\section{CONVERSION OF AREA INTEGRALS TO VOLUME INTEGRALS}

To convert the area integral expressions for the buoyant force and torque into volume integrals we use the differential form version of Stokes' theorem. Let a represent the area of the body, $V$ the volume enciosed by $a$, and use the notation $\partial \mathrm{V}=\mathrm{a}$ to denote the fact that $\mathrm{a}$ is a closed surface bounding $\mathrm{V}$. Stokes' theorem then states that the integral over $\partial \mathrm{V}$ of a two-form $\omega$ is equal to the integral over $\mathrm{V}$ of the exterior derivative $d \wedge \omega$, which is a three-form, namely

$$
\int_{\partial \mathrm{V}} \omega=\int_{\mathrm{V}} \mathrm{d} \wedge \omega .
$$

This is a "modern" version of the divergence theorem. It has the advantage that it is easier to apply to vector-valued integrals. (If the reader feels uncomfortable with this formalism, then all he/she needs to do is write out the area and volume elements in tensor form with the Levi-Civita tensor to handle the area and volume cross products and apply the classic divergence theorem. The differential form version is considerably simpler.)

The vectorial element of surface area is given by the vector valued 2-form

$$
\mathrm{da}_{\mathrm{i}}=\frac{1}{2} \varepsilon_{\mathrm{ijk}} \mathrm{dx} \mathbf{j} \wedge \mathrm{dx}
$$

where we use the convention that repeated indices are summed from one to three and single indices are understood to range from one to three, representing $x, y$, or $z$ components. $\varepsilon_{i j k}$ is the Levi-Civita antisymmetric tensor density with value $\varepsilon_{123}=1$. The 2 -form $\mathrm{dx} j \wedge d x^{k}$ is the area element. The integral of the vector area is zero as can be seen from the computation

$$
\int_{\partial V} d a_{i}=\frac{1}{2} \varepsilon_{i j k} \int_{\partial V} d x^{j} \wedge d x^{k}=\frac{1}{2} \varepsilon_{i j k} \int_{V} d \wedge\left(d x^{j} \wedge d x^{k}\right)=0
$$

where we have used Stokes' theorem in going from the second to the third expression and then used the fact that the exterior derivative of a perfect differential is zero. Note that this does not imply that the area is zero, it only states that the integral of the vector area over a closed surface is zero. 
The buoyant force is given by

$$
F_{B i}=\int_{\partial V}(p 0+\rho g z) d a_{i}=p_{0} \iint_{\partial V} a_{i}+\rho g \frac{1}{2} \varepsilon_{i j k} \int_{\partial V} z d x^{j} \wedge d x^{k} .
$$

The first term vanishes as was shown above. Applying Stokes' theorem to the second term gives the result

$$
\begin{aligned}
F_{B j} & =\rho g \frac{1}{2} \varepsilon_{i j k} \int_{V} d \wedge\left(z d x^{j} \wedge d x^{k}\right) \\
& =\rho g \frac{1}{2} \varepsilon_{i j k} \int_{V} d z \wedge d x^{j} \wedge d x^{k} \\
& =\rho g \delta_{3 i} \int_{V} d x \wedge d y \wedge d z \\
& =\rho g \delta_{3 i} V
\end{aligned}
$$

where $\delta_{\mathrm{ij}}$ is Kronecker's delta tensor that equals one if $\mathrm{i}=\mathrm{j}$ and is zero otherwise. Note that " 3 " denotes the z-direction.

$\Gamma_{\mathrm{Bi}}:$

To determine the center of buoyancy we first evaluate the buoyant torque

$$
\Gamma_{B i}=\rho g \frac{1}{2} \varepsilon_{i j k} \varepsilon_{k l m} \int_{\partial V} X^{j} \mathrm{zdx}^{1} \wedge d x^{m} .
$$

The Levi-Civita tensors satisfy the identity

$$
\varepsilon_{\mathrm{ijk}} \varepsilon_{\mathrm{klm}}=\delta_{\mathrm{il}} \delta_{\mathrm{jm}}-\delta_{\mathrm{im}} \delta_{\mathrm{jl}},
$$

which simplifies the $\Gamma_{\mathrm{Bi}}$ integral to

$$
\Gamma_{B i}=-\rho g \int_{\partial V} z X^{j} d x^{j} \wedge d x^{i} .
$$

Applying Stokes' theorem we find

$$
\begin{gathered}
\Gamma_{B i}=-\rho g \int_{V} d \wedge\left(z X^{j} d x^{j} \wedge d x^{i}\right) \\
=-\rho g \int_{V}\left[X^{j} d z \wedge d x^{j} \wedge d x^{i}+z d \wedge\left(\frac{1}{2} d\left(X^{j} X^{j}\right) \wedge d x^{i}\right)\right] .
\end{gathered}
$$

The second term vanishes because it is the exterior derivative of an exact form. The first term can be written

$$
\Gamma_{B i}=-\rho g \varepsilon_{3 j i} \int_{V} X^{j} d x \wedge d y \wedge d z .
$$

In vector form this becomes

$$
\vec{\Gamma}_{B}=-\rho g \hat{z} \times \int_{V} \vec{X} d^{3} x
$$


The center of buoyancy location vector is then given by

$$
\begin{aligned}
\vec{A}_{\perp} & =\hat{z} \times\left(-\hat{z} \times \frac{1}{v} \int_{V} \vec{X} d^{3} x\right) \\
& =\frac{1}{V} \int_{V} \vec{X} d^{3} x-\hat{z} \frac{1}{V} \int_{V} z d^{3} x,
\end{aligned}
$$

which is just the perpendicular projection of the vector $\frac{1}{v} \int_{V} \vec{X} d^{3} x$ from the $z$ direction (in whic: $\overrightarrow{\mathbf{F}}_{\mathrm{B}}$ points). Even though we can only determine the center of buoyancy to within a projection, we identify the full center of buoyancy location with this vector. 